\section{Consenso de la Sociedad Chilena de Endocrinología y Diabetes sobre resistencia a la insulina}

\author{
FELIPE POLLAK ${ }^{1}$, VERÓNICA ARAYA ${ }^{2}$, \\ ALEJANDRA LANAS ${ }^{2}$, JORGE SAPUNAR ${ }^{3}$
}

COAUTORES (PANEL DE EXPERTOS):

MARCO ARRESE 4 , CARMEN GLORIA AYLWIN 5 , CARMEN GLORIA BEZANILLA ${ }^{6}$, ELENA CARRASCO ${ }^{7}$, FERNANDO CARRASCO ${ }^{8}$, ETHEL CODNER $^{9}$, ERIK DÍAZ $^{10}$, PILAR DURRUTY ${ }^{11}$, JOSÉ GALGANI ${ }^{1}$, HERNÁN GARCÍA ${ }^{12}$, RODOLFO LAHSEN ${ }^{6}$, CLAUDIO LIBERMAN ${ }^{2}$, GLORIA LÓPEZ $^{2}$, ALBERTO MAÍZ ${ }^{1}$, VERÓNICA MUJICA ${ }^{13}$, JAIME PONIACHIK ${ }^{14}$,

TERESA SIR ${ }^{15}$, NÉSTOR SOTO ${ }^{16}$, JUAN VALDERAS ${ }^{1}$, PAULINA VILLASECA ${ }^{17}$, CARLOS ZAVALA ${ }^{6}$

\section{Second Consensus of the Chilean Society of Endocrinology and Diabetes about insulin resistance}

Insulin resistance is a prevalent condition commonly associated with unhealthy lifestyles. It affects several metabolic pathways, increasing risk of abnormalities at different organ levels. Thus, diverse medical specialties should be involved in its diagnosis and treatment. With the purpose of unifying criteria about this condition, a scientific-based consensus was elaborated. A questionnaire including the most important topics such as cardio-metabolic risk, non-alcoholic fatty liver disease and polycystic ovary syndrome, was designed and sent to national experts. When no agreement among them was achieved, the Delphi methodology was applied. The main conclusions reached are that clinical findings are critical for the diagnosis of insulin resistance, not being necessary blood testing. Acquisition of a healthy lifestyle is the most important therapeutic tool. Insulin-sensitizing drugs should be prescribed to individuals at high risk of disease according to clinically validated outcomes. There are specific recommendations for pregnant women, children, adolescents and older people.

(Rev Med Chile 2015; 143: 637-650)

Key words: Intra-abdominal fat; Insulin resistance; Life style; Metformin; Risk factors.
'Departamento de Nutrición, Diabetes y Metabolismo. Facultad de Medicina. Pontificia Universidad Católica de Chile. Santiago, Chile.

²Departamento de Medicina Interna, Sección Endocrinología. Hospital Clínico de la Universidad de Chile. Santiago, Chile.

${ }^{3}$ Departamento de Medicina Interna, Unidad de Endocrinología. Facultad de Medicina. Universidad de la Frontera. Temuco, Chile.

${ }^{4}$ Departamento de Gastroenterología. Facultad de Medicina. Pontificia Universidad Católica de Chile. Santiago, Chile.

${ }^{5}$ Servicio de Medicina, Unidad de Diabetes. Hospital de la Dirección de Previsión de Carabineros de Chile y Facultad de Medicina Universidad de Los Andes. Santiago, Chile.

${ }^{6}$ Departamento de Medicina Interna, Centro de Diabetes, Clínica Las Condes. Santiago, Chile. ${ }^{7}$ Nutricionista. Académico Universidad Autónoma de Chile y Universidad Iberoamericana. Profesor Invitado del Instituto de Nutrición y Tecnología de los Alimentos (INTA), Universidad de Chile. Coordinadora Grupo de Nutrición de la Asociación Latinoamericana de Diabetes (ALAD)

${ }^{8}$ Departamento de Nutrición. Facultad de Medicina. Universidad de Chile. Santiago de Chile.

${ }^{9}$ Instituto de Investigación Materno Infantil. Facultad de Medicina. Universidad de Chile. Santiago de Chile. ${ }^{10}$ Centro de Ejercicio, Metabolismo y Salud.

Departamento de Educación Física. Universidad de la Frontera. Temuco, Chile.

${ }^{11}$ Bioquímico. Facultad de Medicina, Universidad de Chile. Santiago, Chile.

${ }^{12}$ Unidad de Endocrinología Pediátrica, Facultad de Medicina. Pontificia Universidad Católica de Chile. Santiago, Chile.

${ }^{13}$ Servicio Medicina Interna, Hospital Regional de Talca. Universidad Católica del Maule. Talca, Chile.

${ }^{14}$ Departamento de Medicina Interna, Sección Gastroenterología. Hospital Clínico de la Universidad de Chile. Santiago, Chile.

${ }^{15}$ Laboratorio de Endocrinología y Metabolismo del Hospital San Juan de Dios. Facultad de Medicina,

Universidad de Chile. Santiago, Chile.

${ }^{16}$ Servicio de Endocrinología y Diabetes. Hospital San Borja Arriarán. Facultad de Medicina, Universidad de Chile. Santiago, Chile.

${ }^{17}$ Departamento de Endocrinología. Facultad de Medicina. Pontificia Universidad Católica de Chile. Santiago, Chile.

Conflicts of interest: F Pollak received personal fees from Novo Nordisk; Bristol-Myers; Sanofi; and nonfinancial support from Novartis and Jannsen, outside the submitted work. R Lahsen was Medical Advisor for Merck Serono until March 2014. C Liberman provided consultancies to Boehringer Ingelheim (2012-2013) in Linagliptine, in work unrelated to the topic of this publication. CG Ailwyn received personal fees for conferences, advisory boards and attendance to meetings, from Novartis, Roche, Johnson \& Johnson de Chile S.A./Jannsen Chile. The remaining authors had nothing to declare.

Recibido el 8 de abril de 2015, aceptado el 11 de mayo de 2015.

Correspondencia a:

Dr. Felipe Pollak C.

fpollak@med.puc.cl; soched@soched.cl 
E n el año 2008 la Sociedad Chilena de Endocrinología y Diabetes (SOCHED) publicó un primer Consenso sobre Resistencia a la Insulina (RI) y Síndrome Metabólico (SM), con el objetivo de definir conceptos diagnósticos y terapéuticos de este problema ${ }^{1}$. A esa fecha existía confusión entre ambos términos y controversias sobre múltiples aspectos de esta condición, lo que generaba abuso de los métodos de laboratorio para medir la RI, sobrediagnóstico y uso inadecuado de fármacos insulino sensibilizantes.

En opinión de SOCHED, muchos de los aspectos que motivaron su publicación aún están vigentes, a lo que se agrega que cada vez son más las especialidades médicas involucradas en su manejo.

Por estos motivos, se ha elaborado un nuevo consenso, diseñado para responder las preguntas más frecuentes sobre el tema, y que sea una guía para diagnosticar, fijar objetivos de tratamiento y tomar decisiones terapéuticas.

\section{Metodología}

El directorio de SOCHED designó a 4 miembros para coordinar el consenso. Se diseñó un cuestionario, con los aspectos más importantes para la condición en la práctica clínica y las principales controversias sobre diagnóstico y tratamiento. Posteriormente fue enviado a una lista de expertos: académicos universitarios, investigadores activos con publicaciones en el tema y miembros destacados de sociedades científicas.

Para aquellas respuestas en que no se encontró evidencia categórica o no hubo consenso, se diseñó un cuestionario específico con 5 diferentes grados de acuerdo (1. Total desacuerdo, 2. Parcialmente en desacuerdo, 3. Incierto, 4. Parcialmente en acuerdo y 5. Total acuerdo), según metodología Delphi $^{2}$. Este cuestionario se envió al panel de expertos. Se consideró "acuerdo" si al menos 60\% de las respuestas se situaban en la categoría 4 ó 5 .

Finalmente el consenso fue redactado y enviado al directorio de SOCHED para su aprobación. Las conclusiones se resumen en la Tabla 1.

\section{Definición y aspectos generales}

\section{¿Cómo se define la RI?}

Es una condición caracterizada por una menor actividad de la insulina a nivel celular. Se expresa en diferentes vías metabólicas, especialmente a ni- vel del metabolismo glucídico, lipídico y proteico. Los órganos más afectados son hígado, músculo y tejido adiposo, aunque sus repercusiones pueden involucrar a otros sistemas. Como compensación el páncreas aumenta la secreción de la hormona, generando un estado de hiperinsulinismo, Este "hiperinsulinismo compensatorio" es limitado en aquellos individuos con reserva pancreática disminuida, y que presentan hiperglicemia o diabetes mellitus tipo 2 (DM 2). En ellos el nivel de insulinemia puede encontrarse en un rango normal o bajo, pese a la presencia de RI.

La RI puede ser causada por múltiples factores: genéticos (incluyendo severos síndromes monogénicos como leprechaunismo, diabetes lipoatrófica y otros), étnicos, ambientales y secundarios a patologías o fármacos (corticoides y otros); pero también aparece en forma fisiológica en determinadas situaciones de la vida como la adolescencia y desarrollo (por efecto de los esteroides sexuales y de la hormona del crecimiento), embarazo (secundario al lactógeno placentario) y envejecimiento (por sarcopenia y redistribución de la adiposidad).

Discutiremos específicamente sobre la RI "poblacional”, generalmente asociada a la adiposidad visceral, en la cual confluyen factores genéticos y

\section{Tabla 1. Conclusiones del consenso}

1. La RI es una condición fisiopatológica que aumenta el riesgo de eventos cardiometabólicos

2. Es una situación prevalente y se asocia principalmente al exceso de peso y estilo de vida

3. No debe ser considerada una enfermedad

4. Debe diferenciarse del Síndrome Metabólico y de la "prediabetes". Esta última corresponde a glicemia de ayuno alterada o intolerancia a la glucosa

5. El diagnóstico de RI debe basarse en sus manifestaciones (clínicas y/o bioquímicas)

6. Los test de laboratorio para la estimación de la RI deben reservarse a la investigación. No recomendamos su uso rutinario en la práctica clínica

7. La reducción del sobrepeso, la actividad física regular y la alimentación saludable son el pilar del tratamiento

8. La metformina es el fármaco insulino sensibilizante de elección. Además de su indicación en DM 2, puede ser utilizada en Rl, como complemento a las medidas no farmacológicas, cuando existe prediabetes o SOP 
relacionados con nuestro estilo de vida, tales como obesidad, sedentarismo y dieta desequilibrada. En este caso existen alteraciones en la señal insulínica postreceptor y en la secreción de adipoquinas (aumento de TNF- $\alpha$, IL- 6 , angiotensinógeno y PAI- 1 y disminución de adiponectina), lo que genera un estado proinflamatorio ${ }^{3,4}$.

No debe ser confundida con el SM, el cual corresponde a la confluencia en un individuo de factores de riesgo cardiovascular asociados a la RI, en una frecuencia mayor que la atribuible al azar y para el cual existen diversas definiciones ${ }^{5-7}$. El SM presenta una alta correlación con la presencia de RI, pero su ausencia no excluye el diagnóstico de RI.

Tampoco debe ser catalogada como "prediabetes", ya que sólo un porcentaje de los individuos insulino resistentes desarrolla DM 2, incluso en poblaciones de alto riesgo ${ }^{8}$.

\section{¿Es una enfermedad?}

Si bien la RI genera riesgos para la salud, no debe ser considerada una enfermedad, sino una condición fisiopatológica.

\section{¿Cuál es su prevalencia?}

Aproximadamente 25 a 35\% de la población occidental presenta RI.

Dada su asociación con el SM, generalmente se considera su prevalencia como equivalente a la de la RI.

En un estudio nacional realizado en Limache, en 850 individuos de ambos sexos, entre 20 y 28 años de edad, $36 \%$ presentó HOMA-IR alterado y $7 \% \mathrm{SM}^{9}$.

En población pediátrica chilena el 46,2\% de niños con exceso de peso presenta RI y $30 \%$ de los obesos $\mathrm{SM}^{10}$.

En la Encuesta Nacional de Salud del año 2010, el SM estuvo presente en 35\% de los individuos mayores de 15 años, lo que confirma su alta prevalencia $^{11}$.

\section{¿Es la RI un factor de riesgo independiente para desarrollar eventos cardio metabólicos y endocrinos?}

La RI es un factor de riesgo para el desarrollo de DM 2, diabetes gestacional (DMG), dislipidemia aterogénica (triglicéridos elevados, colesterol HDL disminuido y presencia de partículas de LDL pequeñas y densas), hipertensión arterial esencial (HTA) y enfermedad por hígado graso no alcohólico (EHGNA) y se asocia al síndrome de ovario poliquístico (SOP) y de apnea obstructiva del sueño $(\mathrm{SAOS})^{3,12-15}$.

En el SOP tiene un rol controversial, ya que éste se presenta con gran heterogeneidad. Aún así parece influenciar su desarrollo, están fuertemente asociados (33 a 50\% de las mujeres con SOP tiene $\mathrm{SM})$ y cuando ambas condiciones coexisten el síndrome se presenta con características particulares $^{12,16,17}$.

Como factor de riesgo cardiovascular, es difícil determinar si su influencia es independiente o ejerce su efecto a través de la hiperglicemia, HTA y dislipidemia, los que componen el SM. Sin embargo, existe evidencia de que la RI es más frecuente en pacientes con enfermedad coronaria que en la población general, y ella se asocia con inflamación subclínica, lo que favorece la aparición de eventos vasculares $^{18,19}$.

Existen estudios que vinculan a la RI con un aumento del riesgo para algunos cánceres y demencia, sin embargo, es necesaria mayor investigación para definir su rol en estas patologías ${ }^{20-23}$.

\section{¿En qué casos debemos buscar la RI?}

Si bien son múltiples las condiciones clínicas en las que está presente, debe pesquisarse en individuos con factores de riesgo para el desarrollo de DM 2, en sospecha de EHGNA y en mujeres con $\mathrm{SOP}$ en edad fértil. En estos casos el tratamiento específico puede disminuir el riesgo de diabetes, producir mejoría de la histología hepática y aumentar la fertilidad en SOP.

\section{Métodos diagnósticos}

\section{¿Cuáles son los criterios diagnósticos para RI?}

Depende del ámbito de trabajo.

En investigación epidemiológica el diagnóstico se determina de manera relativa. A partir de un valor de insulinemia (o rango de otro test), se establece la RI al compararlo con uno que arbitrariamente se considera normal. Usualmente se elige el percentil 75 (cuartil superior) de la distribución del test en una población como punto de corte para definir su presencia.

En el ámbito clínico, debe basarse en la presencia de manifestaciones características, ya que los resultados de los test de laboratorio son de difícil 
interpretación individual. El uso de estos test se reserva para la investigación, no siendo necesario su uso rutinario en la práctica clínica.

El panel considera manifestaciones (clínicas y bioquímicas) de esta condición:

a. Obesidad abdominal.

b. Hiperglicemia (glicemia alterada en ayuno, intolerancia a la glucosa, DM 2, DMG previa).

c. Acantosis nigricans.

d. Hipertrigliceridemia (triglicéridos plasmáticos $>150 \mathrm{mg} / \mathrm{dl}$ ).

e. Colesterol HDL bajo $(<40 \mathrm{mg} / \mathrm{dl}$ en hombres $\mathrm{y}<50 \mathrm{mg} / \mathrm{dl}$ en mujeres).

f. EHGNA.

g. SOP.

h. HTA esencial.

En relación a ellas:

- La definición de obesidad abdominal está basada en la medición de la circunferencia abdominal. Esta debe realizarse con el paciente de pie, al final de una espiración normal y a nivel de la línea media axilar, en el punto medio entre el reborde costal y la cresta iliaca. Es recomendable realizar al menos 2 mediciones y promediarlas ${ }^{24}$.

- Los puntos de corte de esta medición para nuestra población son controversiales ${ }^{25}$. El National Cholesterol Education Program Treatment Panel III (ATP III) los fija en $\geq 102 \mathrm{~cm}$ en hombres $\mathrm{y} \geq 88 \mathrm{~cm}$. en mujeres, y la Asociación Latinoamericana de Diabetes (ALAD) en su consenso para prediabetes en $\geq 94 \mathrm{~cm}$ en hombres $\mathrm{y} \geq 88 \mathrm{~cm}$ en mujeres ${ }^{25}$. En la reciente guía de "Enfoque de riesgo para la prevención de enfermedades cardiovasculares", el Ministerio de Salud (MINSAL), recomienda los propuestos por la Organización Mundial de la Salud (OMS) que los fija en $\geq 90 \mathrm{~cm}$ para hombres $\mathrm{y} \geq 80 \mathrm{~cm}$ para mujeres (similares al sudeste asiático $)^{26}$. Concordamos con esta recomendación, sin embargo, el panel recalca la necesidad de obtener puntos de corte a nivel nacional.

- La Asociación Americana de Diabetes (ADA) considera a la glicemia alterada en ayuno (100 a $125 \mathrm{mg} / \mathrm{dl}$ ) y a la intolerancia a la glucosa (glicemia de 140 a $199 \mathrm{mg} / \mathrm{dl} 2 \mathrm{~h}$ post carga de glucosa) como "prediabetes". Coincidimos con esta definición ${ }^{27}$.

- La presencia de hiperglicemia (prediabetes,
DM 2 o DMG previa) no debe ser considerada como manifestación de RI si en su etiología existe evidencia o sospecha de causas secundarias (ej. enfermedad pancreática), monogénicas (ej. mutaciones asociadas a MODY) o autoinmunes.

- Similar criterio debe aplicarse a los casos de hipertrigliceridemia de causa genética y de hipertensión arterial secundaria.

- EHGNA comprende esteatosis simple, esteatohepatitis y fibrosis.

La investigación clínica ha buscado cuál asociación de estas manifestaciones se correlaciona de mejor forma con la presencia de RI, describiéndose una alta correlación para circunferencia abdominal con glicemia alterada en ayuno, circunferencia abdominal con hipertrigliceridemia, glicemia alterada en ayuno con hipertrigliceridemia e hipertrigliceridemia con colesterol HDL bajo ${ }^{28-31}$.

\section{¿Cuáles son los test de laboratorio para medir la $R I$ ?}

Los test de laboratorio han sido desarrollados para la investigación clínica y epidemiológica. Pese a ello, su uso en la práctica clínica es habitual.

Existen métodos directos (clamp euglicémico hiperinsulinémico y test de tolerancia a la insulina) e indirectos (modelo mínimo de Bergman, homeostatic model assessment of insulin resistance, medición de insulinemia en ayuno y tests derivados de la prueba de tolerancia a la glucosa oral).

Se considera patrón de oro al clamp, aunque su implementación es compleja y de alto costo ${ }^{32-34}$.

En nuestro medio los más utilizados son la medición de insulinemia en ayuno y postsobrecarga oral a la glucosa (75 g) y el homeostatic model assessment of insulin resistance (HOMA-IR).

En el caso de realizar un test de laboratorio a nivel individual, debe preferirse la medición de insulinemia basal o el cálculo de HOMA-IR ${ }^{35-37}$.

Para insulinemia basal consideramos alterado un valor $\geq 15 \mu \mathrm{UI} / \mathrm{ml}$ y para HOMA-IR sobre $2,6^{38}$.

Ya que la secreción fisiológica de insulina es pulsátil, en ambos test para la medición de insulina basal debiesen tomarse 2 muestras seriadas en un lapso de 10 a $15 \mathrm{~min}$.

En caso de que el resultado sea considerado en rango de RI, debe existir al menos una de las manifestaciones mencionadas para plantear el diagnóstico ${ }^{39,40}$. 
Con respecto a la medición de insulinemia postsobrecarga oral a la glucosa, no lo recomendamos ya que el punto de corte usualmente utilizado $(\geq 60 \mu \mathrm{UI} / \mathrm{ml}$ a las $2 \mathrm{~h}$, en presencia de glicemia $<140 \mathrm{mg} / \mathrm{dl}$ ), proviene de un estudio prospectivo realizado sólo en hombres cuyo objetivo era evaluar riesgo cardiovascular y no ha sido validado en nuestro medio ${ }^{41}$. En un estudio nacional, en que se analizaron 1.800 pruebas de tolerancia oral a la glucosa, realizadas en adultos de ambos sexos, este valor se encontraba en el percentil $55-60^{42}$. Además, como test presenta baja correlación con el clamp y alta variabilidad, lo que puede generar falsos positivos frecuentes ${ }^{1}$.

Debe recalcarse que existen limitantes en la determinación de la insulina. A diferencia de la medición de otras hormonas, no existe un método recomendado internacionalmente. Por otro lado, las técnicas y kits disponibles reaccionan con insulina y con otras moléculas, tales como proinsulina y péptido $C$, las que varían entre individuos. Así, la especificidad difiere significativamente entre las técnicas y kits comerciales. Estas razones impiden definir un punto de corte para insulinemia que sea consistente entre laboratorios de investigación y clínicos. Esta limitante queda de manifiesto en estudios que destacan la escasa comparabilidad entre diferentes técnicas y kits, lo que es corroborado por la $\mathrm{ADA}^{43,44}$. Recomendamos considerar este aspecto y mantener una técnica de medición de insulina y laboratorio específico cuando se realice su seguimiento como objetivo intermedio de tratamiento.

\section{Tratamiento}

\section{¿Cuándo debe ser tratada la RI?}

A excepción de que se presente en situaciones fisiológicas, siempre deben instaurarse medidas para corregir la RI.

Las medidas no farmacológicas ("estilo de vida saludable”) son el pilar del tratamiento.

El uso de fármacos insulino sensibilizantes debe reservarse a aquellas situaciones en que exista alto riesgo de eventos clínicos y su utilidad haya sido demostrada.

Las medidas terapéuticas se resumen en la Figura 1.

\section{¿Cuál es el objetivo del tratamiento?}

Pueden considerarse objetivos intermedios como: reducción de la circunferencia abdominal, reducción de la glicemia plasmática, mejoría del perfil lipídico, reducción de las cifras de presión arterial, mejoría de las pruebas hepáticas (en EHGNA) o de la frecuencia de ovulación (en SOP). Sin embargo, existe suficiente evidencia para recomendar tratamientos dirigidos a la mejoría en desenlaces clínicos, es decir: prevención de DM 2, mejoría de la histología hepática (en EHGNA),

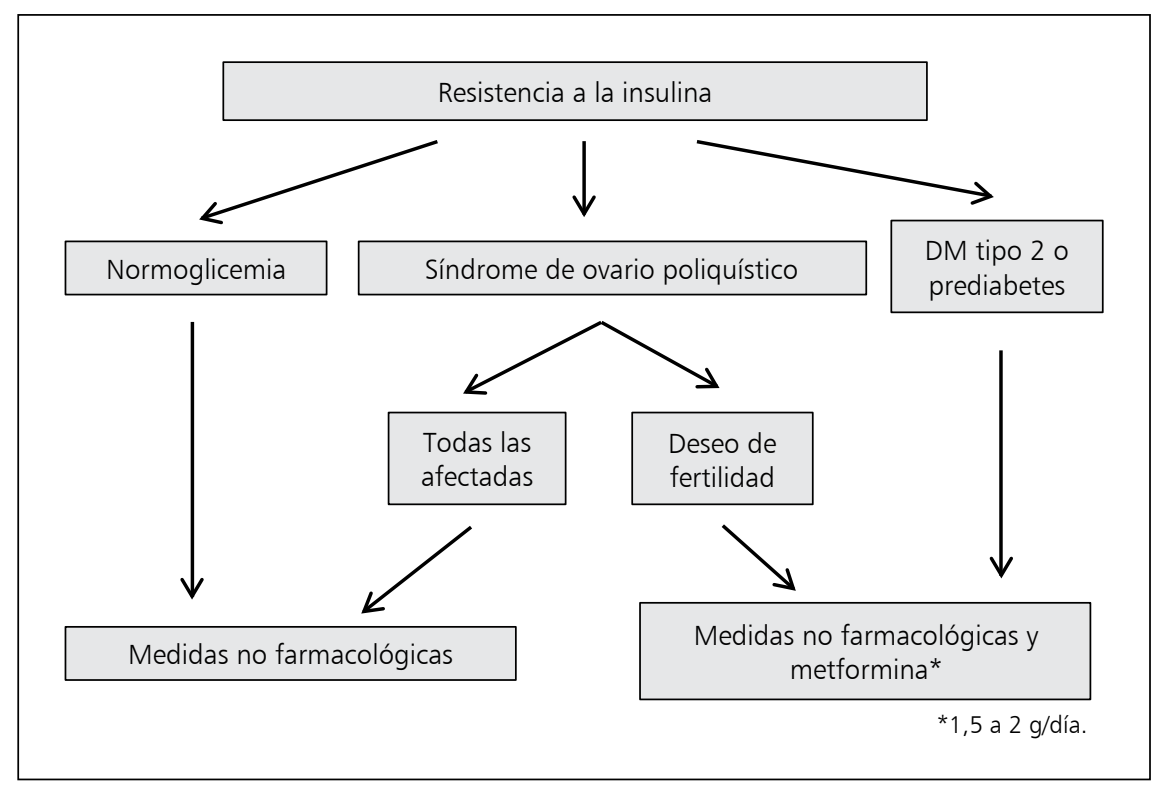

Figura 1. Resumen de medidas terapéuticas. 
mayor número de embarazos (en SOP) y reducción del riesgo cardiovascular en SM.

En este último punto, el tratamiento de la RI sólo ha demostrado reducción de eventos en pacientes con DM 2, debiéndose privilegiar para este objetivo el manejo estricto de todos los factores de riesgo presentes, incluyendo el tabaquismo ${ }^{45}$.

Finalmente, cada patología debe recibir su tratamiento específico según las guías vigentes.

\section{¿Cuáles son las medidas no farmacológicas más importantes?}

El ejercicio físico y la reducción del sobrepeso son las medidas más importantes (recomendación tipo A).

Idealmente deben implementarse en el contexto de un equipo de salud multidisciplinario (médico, nutricionista, kinesiólogo o profesor de educación física).

En los estudios Finish Diabetes Prevention Program (DPS) y US Diabetes Prevention Program (DPP), la actividad física de intensidad moderada en forma regular, una alimentación saludable y la baja de peso de 7\%, disminuyó el riesgo de desarrollo de DM 2 en individuos prediabéticos con RI en aproximadamente 58\%, durante un seguimiento promedio de 3 años ${ }^{46,47}$.

Esta eficacia puede ser menor en la práctica clínica dada la baja adherencia a mediano y largo plazo.

Dado el impacto del ejercicio físico en los parámetros metabólicos, proporcionalmente mayor que la pérdida de peso, es la indicación más importante en individuos normopeso con RI.

\section{¿Cuál es la dieta de mayor beneficio?}

Las recomendaciones nutricionales en pacientes con RI están dirigidas a la corrección del sobrepeso. Se recomienda una pérdida mínima de $5-7 \%$ del peso corporal ${ }^{48}$.

Debe indicarse una dieta balanceada normo o hipocalórica (restricción de 500 a $1.000 \mathrm{Kcal} /$ día de los requerimientos energéticos), dependiendo del estado nutricional del sujeto, con reducción del aporte de grasas saturadas, sacarosa y productos endulzados con fructosa, y un aumento de grasas monoinsaturadas y fibra dietética ${ }^{49,50}$.

Las dietas basadas en un bajo índice glicémico son útiles en objetivos intermedios, pero debe considerarse la complejidad de su aplicación ${ }^{51-53}$.

En estudio recientes, la dieta mediterránea, caracterizada por un alto aporte de frutas, vegetales, cereales, legumbres, frutos secos, pescados $\mathrm{y}$ aceites monoinsaturados, junto a un consumo moderado de vino acompañando las comidas, ha demostrado prevenir la aparición de SM y DM 2 en pacientes con alto riesgo metabólico (independiente de cambios en el peso corporal) y para la prevención primaria de eventos cardiovasculares en individuos de alto riesgo (diabéticos tipo 2 entre ellos), efecto que se extiende al subgrupo de obesidad abdominal ${ }^{54-56}$.

Consideramos que la guía de alimentación del programa de salud cardiovascular del MINSAL cumple con la mayor parte de estas recomendaciones $^{57}$.

\section{¿Cuál es el efecto de la actividad física?}

El ejercicio cumple un rol fundamental mejorando la RI por 2 mecanismos. En la vía dependiente de insulina, restablece la cascada de la señal insulínica al disminuir el efecto deletéreo de los lípidos intramusculares mejorando la oxidación de grasas intramusculares. El segundo mecanismo es mediado por la contracción muscular con activación de AMPK (5' AMP-activated protein kinase). Ambos mecanismos conducen a la traslocación del transportador específico de glucosa GLUT-4. Además, el músculo y páncreas mantienen comunicación mediante mioquinas, lo que permitiría suponer un rol muscular en la secreción de insulina ${ }^{58}$.

Esta mejoría en la sensibilidad a la insulina y utilización de la glucosa tiene alto impacto en la práctica clínica. En el estudio Da Qing, el ejercicio fue el factor más importante en el retardo a la progresión a DM 2, al comparar los grupos de intervención de alimentación (reducción de riesgo de $31 \%$ ) versus actividad física (reducción de riesgo de $46 \%$ ) versus alimentación y ejercicio combinados (reducción de riesgo de 42\%) ${ }^{59}$.

\section{¿Cómo debemos recomendarla?}

En caso de RI la recomendación mínima es practicar ejercicio aeróbico (caminata vigorosa, trote liviano o equivalente), $30 \mathrm{~min} 3$ veces por semana. No deben transcurrir más de 2 días sin realizarla. Idealmente debe llegarse a una mayor frecuencia (150 min semanales) y complementarse con ejercicio anaeróbico (de fuerza). Esta indicación es aproximada a la utilizada en los estudios DPP y DPS. 
Otra opción terapéutica es la indicación de ejercicios de moderada y alta intensidad, en la medida que no produzcan estrés cardiovascular, respiratorio u osteoarticular, pudiendo diseñarse para este fin ciclos de ejercicio de muy corta duración ${ }^{60}$. En esta modalidad han mostrado beneficios:

a. Método de alta intensidad en bicicleta estática: $1 \mathrm{~min}$ de pedaleo con la carga necesaria para inducir la fatiga muscular de las piernas, seguido de recuperación inactiva (sentado en la bicicleta) y repitiendo tal actividad 10 veces. Este esquema de tratamiento se realiza 3 días a la semana.

b. Método de pesas o sobrecarga: empleo de pesas ejercitando grupos musculares aislados. Cada grupo muscular debe llegar a la fatiga muscular realizando flexiones y extensiones en $1 \mathrm{~min}$ de trabajo continuo, luego se descansa y se repite la acción 3 veces (o series). Se realiza 2 veces por semana.

Estas metodologías, con pequeñas variaciones, fueron evaluadas en un estudio nacional con mujeres sedentarias, prediabéticas con sobrepeso u obesidad, durante un período de 12 semanas, obteniéndose mejoría significativa de la glicemia y el HOMA-IR tanto a las 24 como a las $72 \mathrm{~h}$ luego de finalizado cada programa de ejercicio ${ }^{61}$.

La evaluación de la capacidad funcional y la condición física son primordiales para que las cargas de trabajo sean adecuadas a las necesidades terapéuticas de cada paciente ${ }^{62}$.

\section{¿Con qué fármacos contamos para tratar la RI?}

Los fármacos primariamente insulino sensibilizantes son:

a. Metformina: actúa aumentando los niveles de AMPK, disminuye la neoglucogénesis (principal efecto) por inhibición de la glicerofosfato deshidrogenasa a nivel mitocondrial y aumenta la captación muscular de la misma. Otros efectos incluyen disminución de niveles y recambio de ácidos grasos libres, reducción de la obesidad visceral y de los niveles de triglicéridos plasmáticos, mejoría en niveles de factores proinflamatorios y protrombóticos y aumento del efecto incretina. Sus efectos adversos más frecuentes son gastrointestinales, los que varían en frecuencia dependiendo de la formulación y dosis empleada. Se ha descrito una disminución de los niveles de vitamina $B_{12}$, aunque infrecuentemente en rango de deficiencia. Está contraindicada en la insuficiencia renal (clearence de creatinina $<30 \mathrm{ml} / \mathrm{min}$ ) $\mathrm{y}$ en situaciones con riesgo de acidosis láctica ${ }^{63,64}$.

b. Tiazolidinedionas (TZDs): rosiglitazona y pioglitazona, son agonistas de los receptores PPAR-gamma, producen diferenciación del adipocito y redistribución del tejido adiposo, disminuyendo los depósitos viscerales y aumentando los depósitos subcutáneos. Promueven la transcripción de genes involucrados en el metabolismo de carbohidratos, aumentando la síntesis de GLUT- 4 en el músculo y de adiponectina en el adipocito. Los efectos colaterales más importantes son el aumento del peso, la retención hídrica, anemia leve, fracturas óseas (especialmente en mujeres postmenopáusicas) y una aparente mayor incidencia de cáncer de vejiga observada con pioglitazona. El aumento del riesgo cardiovascular en diabéticos tipo 2 con rosiglitazona es controversial. Están contraindicadas en insuficiencia cardiaca y no son recomendadas en osteoporosis ${ }^{64}$.

Existen otros fármacos con acción insulino sensibilizante "indirecta", tales como antidiabéticos (acarbosa, inhibidores de DPP- 4 y agonistas del receptor de GLP-1), para la obesidad (orlistat) y algunos antihipertensivos (IECA, ARA II y doxazosina). Estos fármacos deben reservarse para sus patologías primarias y no ser utilizados específicamente para reducir la RI.

Con respecto a acarbosa debemos mencionar que su uso en prediabéticos redujo la incidencia de DM 2 en un estudio a 3 años, en $25 \%$. Sin embargo, su costo y baja tolerancia gastrointestinal lo hacen poco recomendable para este objetivo ${ }^{65}$.

\section{¿Cuál es el fármaco de elección y cuándo debemos indicarlo?}

Considerando su costo/efectividad y el perfil de reacciones adversas, el fármaco de elección es la metformina.

Su indicación en RI es aceptada como complemento al estilo de vida saludable en presencia de prediabetes (recomendación tipo A), especialmente bajo los 60 años, IMC $\geq 35 \mathrm{~kg} / \mathrm{mt}^{2}$, historia de diabetes en familiares de primer grado, triglicéridos elevados, colesterol HDL disminuido, HTA y hemoglobina glicosilada (HbAlc) $\geq 6,0 \%{ }^{65}$. En el estudio DPP, en menores de 45 años y en obesos severos fue tan eficaz como los cambios intensivos de estilo de vida en la reducción de riesgo para el 
desarrollo de DM 2. No debe ser indicada para la prevención de otros tipos de diabetes.

También se acepta su uso en SOP (ver casos especiales).

De ser indicada debe utilizarse la dosis empleada en los estudios clínicos (1,5 a 2 g al día), pudiendo ser menores en casos de intolerancia digestiva. Es recomendable comenzar con dosis bajas y progresar hasta la dosis terapéutica para obtener una mejor tolerancia.

Si bien su uso resulta razonable para objetivos intermedios en otras situaciones de RI, especialmente cuando el tratamiento no farmacológico no logra controlarlos después de un período de tiempo razonable, sus beneficios clínicos a largo plazo para individuos normoglicémicos no han sido evaluados.

En el caso que las manifestaciones de la RI se hayan controlado y los factores causales sean corregidos (obesidad, sedentarismo), es posible disminuir gradualmente y eventualmente suspender la metformina. Cuando no es así, al igual que las medidas no farmacológicas, el tratamiento debe mantenerse por tiempo indefinido.

El panel es contrario a su uso para promover pérdida de peso.

Con respecto a TZDs, si bien la reducción del riesgo de DM 2 en prediabéticos es similar al estilo de vida saludable, su indicación con este objetivo es discutible debido a su costo y amplio espectro de reacciones adversas ${ }^{66-68}$.

\section{Casos especiales}

\section{a. Adulto mayor (mayores de 65 años)}

\section{¿Cuándo debemos considerar patológica la RI a esta edad?}

La RI es una condición fisiológica asociada a los cambios corporales relacionados con la edad, tales como el aumento de adiposidad central y la sarcopenia. Se debe considerar patológica cuando existan condiciones clínicas que otorguen un mayor riesgo de enfermedades específicas, tales como prediabetes, HTA, EHGNA y enfermedad cardiovascular $^{69-71}$.

\section{Los test que se utilizan en clínica, ¿están validados para este grupo etario?}

Los test diagnósticos no están suficientemente validados en este grupo $^{72}$.
En Chile, un estudio en individuos mayores de 60 años, no diabéticos, describe un valor de HOMA-IR de 2,6 como punto de corte. De ser utilizado, debe analizarse en conjunto con las manifestaciones mencionadas presentes ${ }^{73}$.

\section{¿Cuándo y cómo debemos tratarla?}

Previo a la decisión, debe considerarse el estado general del paciente adoptando una actitud conservadora en aquellos individuos más frágiles.

Dado los cambios propios de la edad se debe fomentar el ejercicio físico, cuyo efecto sobre la insulino sensibilidad es especialmente significativo e independiente de la baja de peso. Se recomienda un programa que alterne ejercicios de fuerza con ejercicios aeróbicos ${ }^{74,75}$.

El régimen es un pilar importante y se debe indicar un aporte calórico adecuado para la mantención o recuperación del estado nutricional. No recomendamos el uso de dietas estrictas o fomentar una pérdida de peso excesiva. Debe recordarse que el IMC normal a esta edad es hasta $27 \mathrm{~kg} / \mathrm{m}^{2}$.

El tratamiento farmacológico no es recomendable en este grupo. En prediabetes su utilización es discutible, ya que en el estudio DPP a diferencia de los cambios en el estilo de vida, el uso de metformina fue ineficaz en prevenir la progresión a la enfermedad en mayores de 60 años ${ }^{47}$.

\section{b. Niños y adolescentes (menores de 18 años)}

\section{¿Cuándo debemos considerar patológica la RI a esta edad?}

No hay consenso al respecto en este grupo etario. Debe tomarse en cuenta que en la adolescencia la condición se presenta en forma fisiológica.

Podemos considerarla patológica cuando se asocia a manifestaciones características y si hay antecedentes de familiares en primer grado con DM 2, bajo peso de nacimiento (PEG) con "catch-up growth" (crecimiento compensatorio) acelerado y pubarquia precoz.

En relación a los puntos de corte para las manifestaciones de RI, deben ser los recomendados para la edad: para circunferencia abdominal debe utilizarse el valor sobre el percentil 90, para triglicéridos sobre el percentil 95 (> $130 \mathrm{mg} / \mathrm{dl}$ para adolescentes), para colesterol HDL bajo el percentil $10(<40 \mathrm{mg} / \mathrm{dl}$ para adolescentes $)$ y para presión arterial sistólica y diastólica sobre el percentil $90^{76,77}$. 


\section{Los test que se utilizan en clínica, ¿están validados para este grupo etario?}

Los test diagnósticos no están validados en este grupo. A esta edad el HOMA-IR tiene una modesta correlación con el clamp y se debe considerar que durante la adolescencia los valores normales de insulina son mayores a lo descrito en adultos, por lo que su utilización puede resultar confundente ${ }^{78}$.

\section{¿Cómo debemos tratarla?}

Se recomienda el estilo de vida saludable. Se debe insistir en estas medidas a pesar de la dificultad en la adherencia. No se recomienda el tratamiento farmacológico a excepción del uso de metformina en pacientes con DM 2 a partir de los 10 años. Otros fármacos insulino sensibilizantes no han sido aprobados para su uso en pediatría ${ }^{79}$.

\section{c. Embarazo}

\section{¿Cuándo debemos considerar patológica la RI en una embarazada?}

La RI es una característica fisiológica del $2^{\circ}$ y $3^{\text {er }}$ trimestre del embarazo y desaparece después del parto.

Es patológica cuando se asocia a condiciones como SOP, prediabetes o DM 2 pregestacional, EHGNA, SM o algunos antecedentes obstétricos como macrosomía, abortos frecuentes, mortinatos, DMG e HTA inducida por el embarazo ${ }^{80}$.

\section{Los test que se utilizan en clínica, ¿están valida- dos en el embarazo?}

Existe un estudio de validación para HOMA-IR en embarazadas obesas, normoglicémicas y sólo para $2^{\circ}$ y $3^{\text {er }}$ trimestre, sin determinación de punto de corte $^{81}$. El clamp se ha utilizado durante el embarazo, pero sólo está indicado con fines de investigación.

En general, para cualquier test no se ha resuelto cuáles son los niveles normales a partir de los cuales se define lo patológico. No recomendamos su utilización, a menos de que se trate de protocolos de investigación.

La presencia de manifestaciones previas es suficiente para el diagnóstico.

\section{¿Cuándo y cómo debemos tratarla? \\ En lo posible, la RI debiese ser diagnosticada y tratada antes del embarazo. Su tratamiento es pre-}

dominantemente no farmacológico, el que además permite controlar que la ganancia de peso durante el embarazo no supere lo recomendable $(12,5 \mathrm{~kg}$ para la paciente de bajo peso, 11,5 kg para la de peso normal y de $7 \mathrm{~kg}$ para sobrepeso u obesa).

La metformina está indicada en dos casos:

a. La paciente se embaraza tomando el fármaco por SOP, prediabetes o DM 2 (diabetes pregestacional).

b. DMG, cuando no se logran glicemias óptimas con las medidas no farmacológicas. Es considerada un fármaco de clase $\mathrm{B}$, no causa daño, pero su seguridad a largo plazo no ha sido demostrada. Es una alternativa al uso de insulina o puede ser usada en combinación con ella. La metformina cruza la barrera placentaria y alcanza en el feto una concentración de $50 \%$ de la de la madre. La tasa de efectos adversos digestivos en los estudios es baja (en menos de $5 \%$ de los casos debe suspenderse y entre 10 y $15 \%$ no alcanza la dosis total indicada por molestias digestivas ${ }^{82,83}$.

Las TZDs son clase C y no están aprobadas en el embarazo.

\section{d. Síndrome ovario poliquístico}

\section{La RI, ¿qué efectos produce en SOP? ¿Debemos considerarla parte del síndrome?}

La RI está presente en un alto porcentaje de las pacientes con SOP, incluso en pacientes sin sobrepeso u obesidad, aunque tiene una fuerte asociación con la obesidad abdominal. Sin embargo, no es un criterio indispensable para el diagnóstico del síndrome.

La RI se relaciona con un aumento de andrógenos de origen ovárico y suprarrenal, y de andrógenos libres por descenso de la SHBG (globulina transportadora de hormonas sexuales) y trastornos ovulatorios y metabólicos, que pueden determinar aumento del riesgo cardiovascular, de DM 2 y de EHGNA ${ }^{16,17}$.

\section{¿Qué beneficios produce su tratamiento?}

El tratamiento de la RI en el SOP es fundamental para el manejo de los aspectos reproductivos (normalización de la ciclicidad ovárica, ovulación, implantación y prevención de DMG) y metabólicos. Por ser el SOP una disfunción endocrino-metabólica crónica, con un fuerte componente 
genético, la intervención terapéutica debe iniciarse precozmente y ser de carácter prolongado.

Las medidas no farmacológicas son la primera indicación en todas las etapas de la vida de la paciente $^{84}$. Los insulinosensibilizantes están indicados en aquellos casos de SOP en mujeres con evidencias clínicas de RI, especialmente en casos de prediabetes o deseo de fertilidad.

\section{¿Cuándo debemos indicar metformina? ¿Por cuánto tiempo?}

En opinión del panel, no existe evidencia que apoye la práctica de indicar metformina en todas las mujeres con SOP desde el diagnóstico. Su indicación debiese reservarse a la presencia de prediabetes o en aquellas mujeres con manifestaciones de RI y que deseen fertilidad, ya que existe evidencia de un aumento del número de embarazos (recomendación tipo B). Puede mantenerse durante todo el embarazo y suspenderse después del parto ${ }^{85,86}$.

$\mathrm{Su}$ efecto sobre un aumento de los nacidos vivos es controversial ${ }^{87}$.

Finalmente, la metformina no ha demostrado beneficios en el tratamiento de las manifestaciones cutáneas del hiperandrogenismo ni en restaurar la ciclicidad ovárica ${ }^{88}$.

\section{e. Enfermedad por hígado graso no alcohólico}

\section{¿Cuál es el rol de la RI en EHGNA?}

Tiene un papel preponderante en su patogenia. Está presente en $90 \%$ de los casos y su severidad se asocia a un mayor compromiso histológico.

La RI se asocia a lipólisis y a un aumento de la entrada de ácidos grasos libres y de síntesis de triglicéridos en los hepatocitos, lo cual desencadena el fenómeno de esteatosis hepática. Paralelamente los mayores niveles de citoquinas proinflamatorias favorecen el daño celular.

\section{Su tratamiento específico, ¿ofrece beneficios en la evolución de la enfermedad?}

En las etapas iniciales de la enfermedad el tratamiento de la RI tiene un papel primordial para evitar la progresión de la enfermedad ${ }^{50}$.

Tanto los tratamientos no farmacológicos como farmacológicos, con insulino sensibilizantes del grupo de las TZDs, han mostrado mejoría significativa en la histología de EHGNA.

\section{Los fármacos insulino sensibilizantes,} ¿disminuyen el daño hepático crónico?

La metformina sólo ha demostrado tener un efecto benéfico temporal en el nivel de transaminasas séricas. La evidencia científica actual, si bien no es muy robusta, señala que las TZDs, específicamente la pioglitazona, han mostrado resultados positivos en el tratamiento de EHGNA, tanto en pruebas hepáticas como en la histología, principalmente en esteatosis e inflamación lobular, aproximadamente en $40 \%$ de los casos. No se ha demostrado claramente una mejoría en la fibrosis hepática. Por los efectos secundarios de este fármaco, especialmente el mayor riesgo de osteoporosis y fracturas, sólo se recomienda en pacientes con alto riesgo de desarrollar enfermedad hepática avanzada $^{89-91}$.

Agradecimientos al Directorio de SOCHED (2014): Dres. Gilberto González V. (Presidente), Néstor Soto I, Jorge Sapunar, Francisco Cordero, Francisca Ugarte, Silvia Acuña, Patricio Davidoff, José Galgani, Marisol García, Francisca Grob, Beatriz Jiménez, Alejandra Lanas, Felipe Pollak, Paulina Silva, Carlos Stehr, Francisco Guarda.

\section{Referencias}

1. López G. Consenso elaborado por la SOCHED sobre Resistencia a la Insulina y Síndrome Metabólico. Aspectos clínicos y terapéuticos. Rev Soc Chil Endocrinol y Diabetes 2008; 4: 272-81.

2. Hasson F, Keeney S, McKenna H. Research guidelines for the Delphi survey technique. J Adv Nurs 2000; 32: 1008-101.

3. Gallager E, Le Roith D, Karnieli E. The metabolic syndrome-from insulin resistance to obesity and diabetes. Endocrinol Metab Clin North Am 2008; 37: 559-79.

4. Mattu H, Randeva H. Role of adipokines in cardiovascular disease. J Endocrinol 2013; 216: 17-36.

5. Third Report of the National Cholesterol Education Program (NCEP) Expert Panel on Detection, Evaluation and Treatment of High Blood Cholesterol in Adults (Adult Treatment Panel III) final report. Circulation 2002; 106: 3143-421.

6. World Health Organization. Report of a WHO Consultation Part I. Definition, diagnosis and classification of diabetes mellitus and its complications. http//whqlibdoc.int/hq/1999/who_ncd_ncs_99.2.pdf.

7. Alberti KG, Zimmet P, Shaw J. The metabolic syndro- 
me-a new worldwide definition. Lancet 2005; 366: 105962.

8. Lillioja S, Mott DM, Spraul M, Ferraro R, Foley JE, Ravussin E, et al. Insulin Resistance and Insulin Secretory Dysfunction as Precursors of Non-Insulin-Dependent Diabetes Mellitus: Prospective Studies of Pima Indians. N Engl J Med 1993; 329: 1988-92.

9. Bustos P, Amigo H, Arteaga A, Acosta AM, Rona RJ. Factores de riesgo de enfermedad cardiovascular en adultos jóvenes. Rev Med Chile 2003; 131: 973-80.

10. Burrows R, Leiva L, Weistaub G, Ceballos X, Gattas $\mathrm{V}$, Lera L, et al. Síndrome metabólico en niños y adolescentes: asociación con sensibilidad insulínica y con magnitud y distribución de la obesidad. Rev Med Chile 2007; 135: 174-81.

11. http://epi.minsal.cl/estudios-y-encuestas-poblacionales/ encuestas-poblacionales/encuesta-nacional-de-salud/ resultados-ens/

12. Bloomgarden ZT. World congress on insulin resistance, diabetes, and cardiovascular disease: Part 1. Diabetes Care 2011; 34 (7): e115-20.

13. Bloomgarden ZT. World congress on insulin resistance, diabetes, and cardiovascular disease: Part 2. Diabetes Care 2011; 34 (8): e126-31.

14. Bloomgarden ZT. World congress on insulin resistance, diabetes, and cardiovascular disease: Part 3. Diabetes Care 2011; 34 (9): e140-5.

15. Bloomgarden ZT. World congress on insulin resistance, diabetes, and cardiovascular disease: Part 4. Diabetes Care 2011; 34 (10): e152-7.

16. Dunaif A. Insulin resistance and the polycystic ovary syndrome: mechanism and implications for pathogenesis. Endocr Rev 1997; 18: 774-800.

17. Setji TL, Brown AJ. Polycystic ovary syndrome: update on diagnosis and treatment. Am J Med 2014; 127 (10): 912-9.

18. Liu J, Grundy SM, Wang W, Smith SC Jr, Vega GL, Wu $\mathrm{Z}$, et al. Ten-year risk of cardiovascular incidence related to diabetes, prediabetes, and the metabolic syndrome. Am Heart J 2007; 153 (4): 552-8.

19. Deveci E, Yesil M, Akinci B, Yesil S, Postaci N, Arikan E, et al. Evaluation of insulin resistance in normoglycemic patients with coronary artery disease. Clin Cardiol 2009; 32 (1): 32-6.

20. Luo Z, Saha AK, Xiang X, Ruderman NB. AMPK, the metabolic syndrome and cancer. Trends Pharmacol Sci 2005; 26 (2): 69-76.

21. Gallagher EJ, LeRoith D. Epidemiology and molecular mechanisms tying obesity, diabetes, and the metabolic syndrome with cancer. Diabetes Care 2013; 36 Suppl 2: S233-9.
22. Raffaitin C, Gin H, Empana JP, Helmer C, Berr C, Tzourio $\mathrm{C}$, et al. Metabolic syndrome and risk for incident Alzheimer's disease or vascular dementia: the Three-City Study. Diabetes Care 2009; 32 (1): 169-74.

23. De la Monte SM. Contributions of brain insulin resistance and deficiency in amyloid-related neurodegeneration in Alzheimer's disease. Drugs. 2012; 1; 72 (1): 49-66.

24. Moreno M. Circunferencia de cintura: una medición importante y útil del riesgo cardiometabólico. Rev Chil Cardiol 2010; 29: 85-7.

25. www.alad-latinoamerica.org/DOCConsenso/PREDIABETES.pdf

26. http://www.redcronicas.cl/wrdprss_minsal/wp-content/uploads/2015/02/RCV-final-modificado-Melanie-15-01-2015.pdf-interactivo-2.pdf

27. American Diabetes Association. Diagnosis and Classification of Diabetes Mellitus. Diabetes Care 2014; 37 (Supplement 1): S81-90.

28. Gennarelli G, Holte J, Berglund L, Berne C, Massobrio $\mathrm{M}$, Lithell $\mathrm{H}$. Prediction models for insulin resistance in the polycystic ovary syndrome. Hum Reprod 2000; 15 (10): 2098-102.

29. George L, Bacha F, Lee S, Tfayli H, Andreatta E, Arslanian S. Surrogate estimates of insulin sensitivity in obese youth along the spectrum of glucose tolerance from normal to prediabetes to diabetes. J Clin Endocrinol Metab 2011; 96 (7): 2136-45.

30. Guerrero-Romero F, Simental-Mendía LE, González-Ortiz M, Martínez-Abundis E, Ramos-Zavala MG, Hernández-González SO, et al. The product of triglycerides and glucose, a simple measure of insulin sensitivity. Comparison with the euglycemic-hyperinsulinemic clamp. J Clin Endocrinol Metab 2010; 95 (7): 3347-51.

31. Giannini C, Santoro N, Caprio S, Kim G, Lartaud D, Shaw M, et al. The triglyceride-to-HDL cholesterol ratio: association with insulin resistance in obese youths of different ethnic backgrounds. Diabetes Care 2011; 34 (8): 1869-74.

32. DeFronzo RA, Tobin JD, Andres R. Glucose clamp technique: a method for quantifying insulin secretion and resistance. Am J Physiol 1979; 237 (3): 214-23.

33. Matsuda $M$, DeFronzo RA. Insulin sensitivity indices obtained from oral glucose tolerance testing: comparison with the euglycemic insulin clamp Diabetes Care 1999; 22 (9): 1462-70.

34. Otten J, Ahrén B, Olsson T. Surrogate measures of insulin sensitivity vs the hyperinsulinaemic-euglycaemic clamp: a meta-analysis. Diabetologia 2014; 57 (9): 17818. 
35. Zavaroni I, Bonini L, Gasparini P, Barilli AL, Zuccarelli A, Dall'Aglio E, et al. Hyperinsulinemia in a normal population as a predictor of non-insulin-dependent diabetes mellitus, hypertension, and coronary heart disease: the Barilla factory revisited. Metabolism 1999; 48 (8): 989-94.

36. Matthews DR, Hosker JP, Rudenski AS, Naylor BA, Treacher DF, Turner RC. Homeostasis model assessment: insulin resistance and beta-cell function from fasting plasma glucose and insulin concentrations in man. Diabetologia 1985; 28 (7): 412-9.

37. Wallace TM, Levy JC, Matthews DR. Use and abuse of HOMA modeling. Diabetes Care 2004; 27 (6): 1487-95.

38. Acosta A, Maíz A, Leighton F, Pollak F, Castilo O. Determinación del índice de resistencia insulínica mediante HOMA en una población de la RM de Chile. Rev Med Chile 2002; 130: 1227-31.

39. Tam CS, Xie W, Johnson WD, Cefalu WT, Redman LM, Ravussin E. Defining insulin resistance from hyperinsulinemic-euglycemic clamps. Diabetes Care 2012; 35 (7): 1605-10.

40. Venkataraman K, Khoo CM, Leow MKS, Khoo EYH, Isaac AV, Zagorodnov V, et al. New Measure of Insulin Sensitivity Predicts Cardiovascular Disease Better than HOMA Estimated Insulin Resistance. PLoS ONE 2013; 8 (9): e74410.

41. Eschwege E, Richard JL, Thibult N, Ducimetière P, Warnet JM, Claude JR, et al. Coronary heart disease mortality in relation with diabetes, blood glucose and plasma insulin levels. The Paris Prospective Study, ten years later. Horm Metab Res 1985; 15: 41-6.

42. Arancibia C, Galgani J, Morales M, Santos J, Valderas JP, Pollak F. Evaluación de la insulinemia post carga oral de glucosa como método diagnóstico de Resistencia a la Insulina. Rev Med Chile 2014; 142 (9): 1106-12.

43. Manley SE, Stratton IM, Clark PM, Luzio SD. Comparison of 11 human insulin assays: implications for clinical investigation and research. Clin Chem 2007; 53 (5): 92232.

44. Staten MA, Stern MP, Miller WG, Steffes MW, Campbell SE. Insulin assay standardization: leading to measures of insulin sensitivity and secretion for practical clinical care. Diabetes Care 2010; 33 (1): 205-6.

45. Uusitupa M, Peltonen M, Lindström J, Aunola S, Ilanne-Parikka P, Valle T, et al. Ten-Year Mortality and Cardiovascular Morbidity in the Finnish Diabetes Prevention Study-Secondary Analysis of the Randomized Trial. PLoS ONE 2009; 4 (5): e5656.

46. Finnish Diabetes Prevention Study Group. Prevention of Type 2 Diabetes Mellitus by Changes in Lifestyle among Subjects with Impaired Glucose Tolerance. N Engl J
Med 2001; 344: 1343-50.

47. Diabetes Prevention Program Research Group. Reduction in the Incidence of Type 2 Diabetes with Lifestyle Intervention or Metformin. N Engl J Med 2002; 346: 393-403.

48. Dietary Guidelines Advisory Commitee (DGAC) on the Dietary Guidelines or Americans 2010. http://www.cnpp.usda.gov/dgas2010-dgacreport.htm

49. Galgani JE, Uauy RD, Aguirre CA, Díaz EO. Effect of the dietary fat quality on insulin sensitivity. Br J Nutr 2008; 100: 471-9.

50. Conlon BA, Beasley JM, Aebersold K, Jhangiani SS, Wylie-Roset J. Nutritional Management of Insulin Resistance in Nonalcoholic Fatty Liver Disease (NAFLD). Nutrients 2013; 5: 4093-114.

51. Arteaga A. El Índice glicémico, una controversia actual. Nutr Hosp (2006) 21 (Supl. 2) 55-60.

52. Krebs JD, Bell D, Hall R, Parry-Strong A, Docherty PD, Clarke $\mathrm{K}$, et al. Improvements in glucose metabolism and insulin sensitivity with a low-carbohydrate diet in obese patients with type 2 diabetes. J Am Coll Nutr 2013; 32 (1): 11-7.

53. Ko BJ, Park KH, Mantzoros CS. Diet patterns, adipokines, and metabolism: where are we and what is next? Metabolism 2014; 63 (2): 168-77.

54. Giuliano D, Exposito K. Mediterranean diet and metabolic disease. Curr Opin Lipidol 2008; 19: 63-8.

55. Salas-Salvadó J, Bulló M, Babio N, Martínez-González MÁ, Ibarrola-Jurado N, Basora J, et al. PREDIMED Study Investigators. Reduction in the incidence of type 2 diabetes with the Mediterranean diet: results of the PREDIMED-Reus nutrition intervention randomized trial. Diabetes Care 2011; 34 (1): 14-9.

56. PREDIMED Study Investigators. Primary Prevention of Cardiovascular Disease with a Mediterranean Diet. N Engl J Med 2013; 368: 1279-90.

57. MINSAL. Manejo alimentario del adulto con sobrepeso u obesidad. web.minsal.cl/portal/.../75fcbd5dc365e5efe04001011f012019.pdf.

58. Egan B, Zierath J. Exercise Metabolism and the Molecular Regulation of Skeletal Muscle Adaptation. Cell Metabolism 2013; 5: 162-84.

59. Li G, Zhang P, Wang J, Gregg EW, Yang W, Gong Q, et al. The long-term effect of lifestyle interventions to prevent diabetes in the China Da Qing Diabetes Prevention Study: a 20-year follow-up study. Lancet 2008; 371 (9626): 1783-9.

60. Gillen JB, Gibala MJ. High intensity interval training: a time-efficient exercise strategy to improve health and fitness. Appl Physiol Nutr Metab 2014; 39 (3): 409-12.

61. Álvarez C, Ramírez R, Flores M, Zúñiga C, Celis-Mo- 
rales CA. Efectos del ejercicio físico de alta intensidad y sobrecarga en parámetros de salud metabólica en mujeres sedentarias, pre-diabéticas con sobrepeso u obesidad. Rev Med Chile 2012; 140: 1289-96.

62. MINSAL. Programa de actividad física para la prevención y control de los factores de riesgo cardiovasculares. web.minsal.cl/portal/.../75fe622727752266e04001011f0169d2.pdf

63. Madiraju AK, Erion DM, Rahimi Y, Zhang XM, Braddock DT, Albright R, et al. Metformin suppresses gluconeogenesis by inhibiting mitochondrial glycerophosphate dehydrogenase. Nature 2014; 26; 510 (7506): 542-6

64. Mizuno CS, Chittiboyina AG, Kurtz TW, Pershadsingh HA, Avery MA. Type 2 Diabetes and Oral Antihyperglycemic Drugs. Current Medicinal Chemistry 2008; 15 : 61-74.

65. Chiasson JL, Josse RG, Gomis R, Hanefeld M, Karasik A, Laakso M. STOP-NIDDM Trail Research Group. Acarbose for prevention of type 2 diabetes mellitus: the STOP-NIDDM randomised trial. Lancet 2002; 15: 359 (9323): 2072-7.

66. Nathan DM, Davidson MB, De Fronzo RA, Heine RJ, Henry RR, Pratley R, et al; American Diabetes Association. Impaired fasting glucose and impaired glucose tolerance: implications for care. Diabetes Care 2007 Mar; 30 (3): 753-9.

67. DREAM (Diabetes REduction Assessment with ramipril and rosiglitazone Medication) Trial Investigators. Effect of rosiglitazone on the frequency of diabetes in patients with impaired glucose tolerance or impaired fasting glucose: a randomised controlled trial. Lancet 2006; 368 (9541): 1096-105.

68. ACT NOW Study. Pioglitazone for Diabetes Prevention in Impaired Glucose Tolerance. N Engl J Med 2011; 364: 1104-15.

69. Dwimartutie N, Setiati S, Oemardi M. The correlation between body fat distribution and insulin resistance in elderly. Acta Med Indones 2010; 42 (2): 66-73.

70. Krentz AJ, Viljoen A, Sinclair A. Insulin resistance: a risk marker for disease and disability in the older person. Diabet Med 2013; 30: 535-48.

71. Kohrt WM, Kirwan JP, Staten MA, Bourey RE, King DS, Holloszy JO. Insulin Resistance in Aging Is Related to Abdominal Obesity. Diabetes 1993; 42: 273-81.

72. Chang AM, Smith MJ, Bloem CJ, Galecki AT, Halter JB, Supiano MA. Limitation of the Homeostasis Model Assessment to Predict Insulin Resistance and B-Cell Dysfunction in Older People. J Clin Endocrinol Metab 2006; 91: 629-34.

73. Garmendia ML, Lera L, Sánchez H, Uauy R, Albala A.
Valores normativos de resistencia a la insulina mediante HOMA-IR en adultos mayores de Santiago de Chile. Rev Med Chile 2009; 137: 1409-16.

74. Kodama S, Shu M, Saito K, Murakami H, Tanaka K, Kuno S, et al. Even low-intensity and low-volume exercise training may improve insulin resistance in the elderly. Intern Med 2007; 46 (14): 1071-7.

75. Coker RH, Hays NP, Williams RH, Brown AD, Freeling SA, Kortebein PM, et al. Exercise-Induced Changes in Insulin Action and Glycogen Metabolism in Elderly Adults. Med Sci Sports Exerc. 2006; 38 (3): 433-8.

76. Fernández JR, Reeden DT, Petrobielli A, Allison DB. Waist circunference percentiles in nationally representative sample of african-american, european-american and mexican american children and adolescent. J Pediatr 2004; 145: 439-44.

77. Barja S, Cordero M, Baeza C, Hodgson M. Diagnóstico y tratamiento de las dislipidemias en niños y adolescentes. Recomendaciones de la Rama de Nutrición de la Sociedad Chilena de Pediatría. Rev Chil Pediatr 2014; 85 (3): 367-77.

78. Schwartz B, Jacobs DR Jr, Moran A, Steinberger J, Hong $\mathrm{CP}$, Sinaiko AR. Measurement of insulin sensitivity in children: comparison between the euglycemic-hyperinsulinemic clamp and surrogate measures. Diabetes Care 2008; 31 (4): 783-8.

79. Levy-Marchal C, Arslanian S, Cutfield W, Sinaiko A, Druet $C$, Marcovecchio $M$, et al. Insulin resistance in children: consensus, perspective, and future directions. J Clin Endocrinol Metab 2010; 95: 5189-98.

80. Catalano PM. Obesity, insulin resistance, and pregnancy outcome. Reproduction 2010; 140: 365-71.

81. Cohen O, Epstein GS, Weisz B, Homko CJ, Sivan E. Longitudinal assessment of insulin sensitivity in pregnancy. Validation of the homeostasis model assessment. Clinical Endocrinology 2006; 64, 640-4.

82. Rowan JA, Hague WM, Gao W, Battin MR, Moore MP. MiG Trial Investigators. Metformin versus Insulin for the Treatment of Gestational Diabetes. N Engl J Med 2008; 358: 2003-25.

83. Holt RI, Lamber KD. The use of oral hypoglycaemic agents in pregnancy Diabet Med 2014; 31: 282-91.

84. Domecq JP, Prutsky G, Mullan RJ, Hazem A, Sundaresh $\mathrm{V}$, Elamin $\mathrm{MB}$, et al. Lifestyle modification programs in polycystic ovary syndrome: systematic review and meta-analysis. JCEM 2013; 98: 4655-63.

85. Moll E, van der Veen F, van Wely M. The role of metformin in polycystic ovary syndrome: a systematic review. Hum Reprod Update 2007; 13: 527-37.

86. Vanky E, Stridsklev S, Heimstad R, Romundstad P, Skogøy K, Kleggetveit O, et al. Metformin versus pla- 
cebo from first trimester to delivery in polycystic ovary syndrome: a randomized, controlled multicenter study. JCEM 2010; 95: E448-455.

87. Palomba S, Falbo A, Orio F Jr, Zullo F. Effect of preconceptional metformin on abortion risk in polycystic ovary syndrome: a systematic review and meta-analysis of randomized controlled trials. Fertil Steril 2009; 92: 1646-58.

88. Tang T, Lord JM, Norman RJ, Yasmin E, Balen AH. Insulin-sensitising drugs (metformin, rosiglitazone, pioglitazone, D-chiro-inositol) for women with polycystic ovary syndrome, oligo amenorrhoea and subfertility.
Cochrane Database Syst Rev 2010;1:CD003053.

89. Ozturk ZA, Kadayifci A. Insulin sensitizers for the treatment of non-alcoholic fatty liver disease. World J Hepatol 2014; 6 (4): 199-206.

90. Boettcher E, Csako G, Pucino F, Wesley R, Loomba R. Meta-analysis: pioglitazone improves liver histology and fibrosis in patients with non-alcoholic steatohepatitis. Aliment Pharmacol Ther 2012; 35: 66-75.

91. Arab JP, Candia R, Zapata R, Muñoz C, Arancibia JP, Poniachik J, et al. Management of nonalcoholic fatty liver disease: an evidence-based clinical practice review. World J Gastroenterol 2014; 20 (33). 